Hirokazu Tanaka, Terrence J. Sejnowski and John W. Krakauer

J Neurophysiol 102:2921-2932, 2009. First published Sep 9, 2009; doi:10.1152/jn.90834.2008

You might find this additional information useful...

Supplemental material for this article can be found at:

http://jn.physiology.org/cgi/content/full/90834.2008/DC1

This article cites 51 articles, 28 of which you can access free at: http://jn.physiology.org/cgi/content/full/102/5/2921\#BIBL

Updated information and services including high-resolution figures, can be found at: http://jn.physiology.org/cgi/content/full/102/5/2921

Additional material and information about Journal of Neurophysiology can be found at: http://www.the-aps.org/publications/jn

This information is current as of November 15, 2009. 


\title{
Adaptation to Visuomotor Rotation Through Interaction Between Posterior Parietal and Motor Cortical Areas
}

\author{
Hirokazu Tanaka, ${ }^{1,3}$ Terrence J. Sejnowski, ${ }^{1,4}$ and John W. Krakauer ${ }^{2}$ \\ ${ }^{1}$ Howard Hughes Medical Institute, Computational Neurobiology Laboratory, The Salk Institute for Biological Studies, La Jolla, \\ California; ${ }^{2}$ Motor Performance Laboratory, Department of Neurology, Columbia University College of Physicians and Surgeons, New \\ York City, New York; ${ }^{3}$ National Institute of Information and Communications Technology (NiCT), Kyoto, Japan; ${ }^{4}$ Division of Biological \\ Sciences, University of California at San Diego, La Jolla, California
}

Submitted 30 July 2009; accepted in final form 2 September 2009

\begin{abstract}
Tanaka H, Sejnowski TJ, Krakauer JW. Adaptation to visuomotor rotation through interaction between posterior parietal and motor cortical areas. J Neurophysiol 102: 2921-2932, 2009. First published September 9, 2009; doi:10.1152/jn.90834.2008. Studying how motor adaptation to visuomotor rotation for one reach direction generalizes to other reach directions can provide insight into how visuomotor maps are represented and learned in the brain. Previous psychophysical studies have concluded that postadaptation generalization is restricted to a narrow range of directions around the training direction. A population-coding model that updates the weights between narrow Gaussian-tuned visual units and motor units on each trial reproduced experimental trial-by-trial learning curves for rotation adaptation and the generalization function measured postadaptation. These results suggest that the neurons involved in rotation adaptation have a relatively narrow directional tuning width $\left(\sim 23^{\circ}\right)$. Population coding models with units having broader tuning functions (such as cosine tuning in motor cortex and Gaussian sum in the cerebellum) could not reproduce the narrow single-peaked generalization pattern. Visually selective neurons with narrow Gaussian tuning curves have been identified in posterior parietal cortex, making it a possible site of adaptation to visuomotor rotation. We propose that rotation adaptation proceeds through changes in synaptic weights between neurons in posterior parietal cortex and motor cortex driven by a prediction error computed by the cerebellum.
\end{abstract}

\section{N T R O D U C T I O N}

The question explored here is how training or errors experienced in one part of the workspace generalize to other parts of the workspace during motor adaptation. Measures of generalization to visuomotor transformations can provide insight into how the learned mapping is represented in the brain (Poggio and Bizzi 2004; Shadmehr 2004). One measure is to probe how a fully learned local remapping generalizes to other unlearned locations (postadaptation generalization) (Ghahramani et al. 1996; Imamizu et al. 1995; Krakauer et al. 2000; Malfait et al. 2002; Mattar and Ostry 2007). Alternatively, trial-by-trial generalization can be assessed during adaptation by examining how errors experienced on trial $k$ transfer to another part of the workspace in trial $k+1$ using a single-state state-space model (Donchin et al. 2003; Shadmehr 2004; Thoroughman and Shadmehr 2000).

This paper focuses on a physiological model of rotation learning that accounts for both trial-by-trial generalization and

Address for reprint requests and other correspondence: H. Tanaka, National Institute of Information and Communications Technology (NiCT), Hikaridai 2-2-2, Keihanna Science City Kyoto 619-0288, Japan (E-mail: hirokazu @ salk.edu). postadaptation generalization. Despite many psychophysical studies of visuomotor rotation, the underlying physiological mechanisms and neural substrates for this type of motor learning remain largely unknown. We derived a physiologically plausible computational model of rotation learning based on population coding and a gradient-descent learning rule. The dependence of adaptation rate on target number, the postadaptation generalization function width, and the trial-by-trial generalization function width were reproduced in a model in which the units were narrowly tuned to their preferred target directions but not when the units were broadly tuned.

Physiological, imaging, and clinical studies have suggested that several brain areas are involved in visuomotor rotation, including the cerebellum (Della-Maggiore and McIntosh 2005; Krakauer et al. 2004; Tseng et al. 2007), ventral premotor cortex (Krakauer et al. 2004), primary motor cortex (HadipourNiktarash et al. 2007; Paz and Vaadia 2004, Paz et al. 2003; Wise et al. 1998), and posterior parietal cortex (Diedrichsen et al. 2005; Inoue et al. 2000; Krakauer et al. 2004). The model made predictions for the functions that are carried out in these brain areas during adaptation to visuomotor rotation and how the visuomotor remapping might be neurally encoded. In addition, the model provided a new explanation and interpretation for results from recent studies reporting selective neural activity enhancement in a particular population of neurons in primary motor cortex (M1) after learning visuomotor rotations (Paz and Vaadia 2004; Paz et al. 2003).

\section{METHOD S}

\section{Participants}

Twelve right-handed participants (age: $27.7 \pm 7.6$ ) volunteered to participate in the experiment. They signed an institutionally approved consent, were unaware of the nature of the experiment, and were paid to participate.

\section{Experimental procedure}

POSTADAPTATION GENERALIZATION. To allow comparison with model simulations, we briefly describe the experimental procedure used in our previous publication, which investigated rotation adaptation and postadaptation generalization (Krakauer et al. 2000). Participants sat in front of a computer screen and controlled a screen cursor using a hand-held indicator. Vision of the arm and hand was blocked with an opaque shield. Participants were asked to move a screen cursor from a common starting point to eight circular targets arrayed in a circle and displayed on a vertical screen. Targets were always 
visible on the screen and were presented in a pseudorandom order (each target was presented once in 8 consecutive movements, and the same target was not presented on successive trials). There was continuous visual cursor feedback during training. Participants were instructed to make straight and uncorrected out-and-back movements with a sharp reversal in the target. In the training block, participants adapted to a $30^{\circ}$ counterclockwise $(\mathrm{CCW})$ rotation. After adaptation had approached asymptote, generalization across directions was measured in the testing block by having participants reach to untrained targets in the absence of visual feedback (postadaptation generalization). To investigate the effect of training target number on learning rate and generalization, participants were trained with single, two, four, or eight targets. Directional error was measured as the angular difference in degrees between the target and the cursor at the position of peak velocity.

TRIAL-BY-TRIAL GENERALIZATION. The setup was the same as in our previous research (Krakauer et al. 2000) with the following exceptions: Participants were only trained to eight targets; target presentation was random rather than pseudorandom; and visual feedback was always present (no test probes). Directional error data were used to evaluate the impact of error on the $k$ th trial on the subsequent $(k+1)$ th trial using the single-state state-space approach described in the following text. The truly random target order, which allowed consecutive movements to the same target, provided the sampling of all possible angular separations between consecutive trials needed to evaluate trial-by-trial generalization using the single-state model. Participants made $\leq 264$ movement trials under visuomotor rotation, and all were presented with the same target order. Movement errors were measured in degrees and trials with directional errors larger than $35^{\circ}$ were excluded from further analyses. The rejected trials constituted only $2.0 \%$ of all trials on average, and only $5.7 \%$ for the worst participant.

\section{Analysis of trial-by-trial generalization using a single-state state-space model}

To assess how an error experienced in one direction influenced learning in another direction, we used the single-state state-space model originally introduced for force-field adaptation (Donchin et al. 2003; Thoroughman and Shadmehr 2000). Here we recast the single-state analysis into a form that is applicable to any form of motor adaptation, including visuomotor rotation learning. The analysis begins by defining a state-space model that consists of a state vector, which is usually not directly observable, and an observable variable.

The state-space model has an eight-dimensional state vector $\Phi$ and an observable scalar $\phi$. The $p$ th component of vector $\Phi, \phi^{(p)}$, denotes the hand-movement direction in degrees when the target $p(P=1, \cdots, 8)$ is shown. The observable scalar $\phi$, which is one component of $\Phi$, is actually the performed hand-movement direction in degrees. The equations that define the state-space model are

$$
\begin{gathered}
\Phi_{k+1}=\Phi_{k}+B H_{k}^{\mathrm{T}} \Delta \phi_{k} \\
\phi_{k}=H_{k} \Phi_{k}
\end{gathered}
$$

The first equation describes how an error $\Delta \phi_{k}$ in the $k$ th trial updates the current state vector $\Phi_{k}$ to the next state $\Phi_{k+1}$, and the second equation describes which component of the state vector is actually observed. Here note that the subscripts $k$ represent the trial number and that the superscripts in parentheses $(p)$ the target directions. Although others have developed state-space models based on a vectorial error (Donchin et al. 2003), we used instead the scalar angular error measured experimentally. To assess trialby-trial generalization, this single-state model was fit either to the experimental trial error sequence or to artificial trial error sequence generated by the population coding model developed in the text below.

PARAMETER ESTIMATION. The matrix $B$ in $E q .1$ represents how the angular error experienced in the current trial updates the state vector and thus defines trial-by-trial generalization. While $B$ is an $8 \times 8$ matrix with 64 components, we imposed a symmetry condition to reduce the number of adjustable parameters to avoid over-fitting to the experimental data. We assumed rotational symmetry, that is, the degree of trial-by-trial generalization depends only on the angular difference between the current and subsequent direction. With this symmetry assumption, the matrix $B$ can be described with eight adjustable parameters: extent of trial-by-trial generalization between targets separated by $-135,-90,-45,0,45,90,135$, and $180^{\circ}$.

Ideally, before rotation learning, the motor output direction $\phi^{(p)}$ for the $p$ th target should be the same as the visually perceived target direction $\theta^{(p)}$. We found, however, that there were small biases from the visually perceived target direction, which varied from participant to participant and led to differences between participants' error time course. To take these biases into consideration, we optimized the initial condition of $\Phi$. Thus there were a total of 16 adjustable parameters: 8 describing matrix $B$ and 8 determining the initial condition $\Phi_{1}$ of $\Phi$. Once the parameters and target order were given, the state-space model produced a trial-by-trial error sequence $\left\{\Delta \phi_{\mathrm{k}}^{\text {trial-analysis }}\right\}$, and we looked for the parameter value that best explained the data. In the prediction-error method (Goodwin and Sin 1984), the 16 parameters $\lambda \equiv\left(B, \Phi_{1}\right)$ were optimized so as to minimize the quadratic error

$$
\hat{\lambda}=\operatorname{argmin}_{\lambda} \sum_{\text {k:trial number }}\left(\Delta \phi_{k}^{\text {data }}-\Delta \phi_{k}^{\text {trial-analysis }}\right)^{2}
$$

Here the error time course $\left\{\Delta \phi_{\mathrm{k}}^{\text {data }}\right\}$ may either be experimentally derived or generated artificially with the population-coding model that we introduce in the following text. We used the downhill simplex method (or the Nelder-Mead method) (Press et al. 1992) because the optimization problem was nonlinear and the derivative with regard to the parameters was not easy to obtain. We first iterated the optimization procedure from randomized initial conditions to confirm that the optimized parameters were global minima. We then calculated the trial-by-trial error generalization function that evaluates how a directional error observed in one direction, trial $k$, influenced a handmovement direction in the next trial, $k+1$. To assess how dependable the optimized parameters were, we performed a standard bootstrapping analysis to evaluate their confidence intervals (Efron 1982). The computation outlined in the preceding text was iterated to obtain each participant's optimized parameters and confidence intervals using 200 bootstrap samples. We confirmed that a further increase of bootstrap samples for each participant did not significantly affect the results. The maximum-likelihood estimator (MLE) was

$$
B=\frac{\sum_{i} \sigma_{i}^{-2} B_{i}}{\sum_{i} \sigma_{i}^{-2}}
$$

where $B_{i}$ and $\sigma_{i}$ are the degree of generalization and its SD of $i$ th participant, respectively, that were evaluated using the bootstrap method. The MLE is optimal in the sense of producing the minimumvariance estimate, under the assumption that $B_{i}$ 's are independent Gaussian variables with variance $\sigma_{i}^{2}$. The variance of the weighted average is obtained as $1 / \Sigma_{i} \sigma_{i}^{-2}$. The weighted average across participants was necessary to obtain a reliable result.

The single state-space (Eqs. 1 and 2) is a linear, deterministic approximation for rotation adaptation, and nonlinear effects that cannot be modeled by the linear state-space model contribute as random noise. We applied the prediction-error method to artificially generated error data with various noise levels and tested whether this estimation method could reproduce the parameter values (see supple- 
mentary materials ${ }^{1}$ for details). Our numerical results confirmed the validity of the prediction-error method even under the influence of random noise.

Maximum likelihood (ML) methods for estimating state-space model parameters have been developed and shown to have some theoretical advantages as an alternative to the prediction-error method described in the preceding text (Cheng and Sabes 2006; Shumway and Stoffer 1982) (see also DISCUSSION). Because ML solutions are usually analytically intractable, solutions are often obtained using an iterative procedure known as the expectation-maximization (EM) algorithm. To assess its performance, we applied the EM algorithm to artificial trial errors that were created with a single-state state-space model with a known parameter set and investigated whether the EM algorithm could recover that parameter set, starting with various initial conditions. We found that parameter estimation using the EM algorithm was highly sensitive to the initial values assigned to the parameters. This probably resulted from the relatively high dimensionality of the state-space model ( 8 dimensions). Consequently, we used the prediction-error method, which is numerically more robust, throughout this study.

\section{Population-coding model for visuomotor rotation learning}

The goal of the population-coding model was to account for visuomotor rotation generalization data using distributed representations of direction tuning similar to those found in various brain regions. The model converted a target direction $(\theta)$ into a handmovement vector $(\vec{r})$. We chose the hand-movement vector as the output variable of the model based on previous studies showing that learning of a rotated reference frame involves remapping a movement vector (Krakauer et al. 2000; Wang and Sainburg 2005). The model posits computational units that are tuned to target direction $\left(\left\{\theta_{i}\right\}\right)$ and hand-movement direction $\left(\left\{\vec{d}_{i}\right\}\right)$. We assumed that the $\theta_{i}$ 's are evenly distributed over all directions. Because the tuning of these units has a finite width, the hand-movement direction cannot be uniquely determined from a single-unit's activity. Rather, the hand-movement direction is determined by summing up all the units' activities multiplied by their preferred directions

$$
\vec{r}=\vec{r}(\theta)=\sum_{i=1}^{N} \vec{d}_{i} g_{i}(\theta)
$$

where there are $N$ computational units each assumed to have same tuning function that differ only in their preferred directions

$$
g_{i}(\theta)=g\left(\theta-\theta_{\mathrm{i}}\right)
$$

where $\theta$ is the angular target direction and $\left\{\vec{d}_{i}\right\}$ are the weight vectors. Here we only considered reaching movements restricted to the horizontal plane, and all the vectors were accordingly two dimensional. The tuning function $g(\theta)$ was made periodic by imposing $\mathrm{g}\left(360^{\circ}\right)=$ $g(0)$. We assumed that the mapping of a visually perceived direction $\theta$ onto a hand-movement vector $\vec{r}$ was purely feedforward given that in the experiments the participants were instructed to perform fast out-and-back movements without on-line corrections (Krakauer et al. 2000). Similar network models have previously been proposed for computing hand-movement direction from a population of M1 cells (Georgopoulos et al. 1982; Schwartz 1994; Taylor et al. 2002), for transforming coordinate systems (Pouget and Sejnowski 1997; Salinas and Abbott 1995), and for force-field motor adaptation (Donchin et al. 2003; Thoroughman and Shadmehr 2000).

We assumed that before learning the rotated remapping, the coordinate transformation was well calibrated from extensive experience of reaching under conditions of normal visual feedback in which the visually perceived and actual hand-movement directions were the same. Initially, the weight vectors $\left\{\vec{d}_{i}\right\}$ were determined in such a way

\footnotetext{
${ }^{1}$ The online version of this article contains supplemental data.
}

that the squared error was minimized between a hand-movement vector $\vec{r}(\theta)$ generated by the model with given visual direction $\theta$ and a visually perceived target vector $\vec{r}=(\cos \theta, \sin \theta)^{T}$

$$
\varepsilon=\frac{1}{2}(\Delta \vec{r})^{2}=\frac{1}{2}\left(\vec{r}_{\mathrm{i}}-\vec{r}\right)^{2}
$$

Because only the movement direction and not the movement amplitude was of interest, we used a unit-length vector for a target while the weight vectors were being trained. This error was minimized over all possible target directions by straightforwardly computing the weight vectors using the standard methods used for radial basis functions (Bishop 1995).

During visuomotor rotation learning, the screen cursor direction was rotated counter-clockwise by $30^{\circ}$ with respect to the actual hand-movement direction. Because the task was to learn the rotated remapping of the movement vector, the cost function to be minimized was the squared error between the clockwise rotated target direction and the actual movement direction

$$
\varepsilon=\frac{1}{2}(\Delta \vec{r})^{2}=\frac{1}{2}\left(R^{T} \vec{r}_{i}-\vec{r}\right)^{2}
$$

where the matrix $R$ represents a $30^{\circ} \mathrm{CCW}$ rotation (and $R^{T}$ represents $30^{\circ} \mathrm{CW}$ rotation). We assumed that the weight vectors were modified during rotation adaptation. To decrease the squared error, the weight vectors were changed by steepest gradient descent

$$
\Delta \vec{d}_{i}=-\eta \frac{\partial \varepsilon}{\partial \vec{d}_{i}}=\eta g_{i}(\theta) \Delta \vec{r}
$$

where $\eta$ is a constant learning rate that was matched to the learning speed found in the experiment. This learning rule involved the rotation matrix, which could be obtained by comparing the movement predicted by the forward model and the actual movement (see DIScusSION).

In every trial, the model reported a vectorial error, $\Delta \vec{r}$, and the vector weights were updated accordingly. As the weights were updated, the model gradually learned the rotated remapping. Given a specific target order, the model was able to predict a corresponding trial-by-trial error time course in a vector representation. In the psychophysical experiments, the error was measured not as a vector representation but by a scalar, i.e., as an angular difference between the rotated cursor direction and the target direction, as in previous studies (Krakauer et al. 2000). We therefore transformed the vectorial error generated by the model into an angular error to facilitate comparison with experimental results. The time course of error reduction showed inter-participant variability even when the target order was the same for all participants. To simulate this variability, we added a two-dimensional noise vector to the output vector $\vec{r}$ distributed as two-dimensional Gaussian.

GENERALIZATION. We considered how errors in one direction influenced errors in other directions, as studied previously with forcefield adaptation (Donchin et al. 2003; Thoroughman and Shadmehr 2000). To better understand how the tuning function shape determines trial-by-trial generalization, we introduce matrix notation

$$
\vec{r}=\sum_{i=1}^{N} \vec{d}_{i} g_{i}(\theta)=\mathbf{D g}(\theta)
$$

where $\mathbf{D}$ is an $2 \times N$ matrix composed of weight vectors, $\mathbf{D}=\left(\vec{d}_{1}\right.$, $\left.\vec{d}_{2}, \cdots, \vec{d}_{N}\right)$, and $\mathbf{g}$ is a $N$-dimensional vector composed of units' activities, $\mathbf{g}(\theta)=\left[g_{1}(\theta), g_{2}(\theta), \cdots, g_{N}(\theta)\right]^{T}$. Then the learning rule becomes 


$$
\Delta \mathbf{D}=\eta \Delta \vec{r} \mathbf{g}^{T}(\theta)
$$

Assume that the $k$ th movement in direction $\theta^{(k)}$ is made with error $\Delta \vec{r}^{(k)}$. Then according to the learning rule

$$
\mathbf{D}^{(k+1)}=\mathbf{D}^{(k)}+\Delta \mathbf{D}^{(k)}=\mathbf{D}^{(k)}+\eta \Delta \vec{r}^{(k)} \mathbf{g}^{T}\left(\theta^{(k)}\right)
$$

where the superscripts on the variables denote the trial number. This modification, in turn, changes the hand-movement vector for the $(k+$ 1)th trial in direction $\theta^{(k+1)}$ as

$$
\begin{aligned}
\mathbf{D}^{(k+1)} \mathbf{g}\left(\theta^{(k+1)}\right)-\mathbf{D}^{(k)} \mathbf{g}\left(\theta^{(k+1)}\right)=\Delta \mathbf{D}^{(k+1)} \mathbf{g}\left(\theta^{(k+1)}\right) & \\
& =\eta \mathbf{g}^{T}\left(\theta^{(k)}\right) \mathbf{g}\left(\theta^{(k+1)}\right) \Delta \vec{r}^{(k)}
\end{aligned}
$$

Thus the degree to which error in one direction influences error in another direction, $\theta^{(k+1)}$, defined as trial-by-trial generalization, depends linearly on the overlap of activities, $\mathbf{g}^{T}\left(\theta^{(k)}\right) \mathbf{g}\left(\theta^{(k+1)}\right)$. The degree of generalization to other directions is dependent on the shape of the tuning function but not on the weight vectors. This is a desirable property because the tuning function shape can be measured physiologically but not the weight vectors. It is therefore possible, if the model is correct, to attribute a neuronal tuning profile to generalization data without knowing the weight vectors. Our analysis of generalization in fact does not depend on the exact number of units because, if $N$ is large enough, the overlap of activity $\mathbf{g}^{T}\left(\theta^{(k)}\right) \mathbf{g}\left(\theta^{(k+1)}\right)$ in $E q .13$ can be approximated by an integral

$$
\frac{N}{2 \pi} \int_{-\pi}^{\pi} d \theta g\left(\theta^{(k)}-\theta\right) g\left(\theta^{(k+1)}-\theta\right)
$$

and $N$ can be absorbed by renormalizing the learning rate $\eta$ so that the degree of generalization to other directions becomes independent of $N$. This is also desirable because it is impossible, in a physiological experiment, to enumerate all the neurons that are involved in computing a visuomotor transformation. Population modeling of trial-bytrial generalization examines how weight vectors change during rotation learning, whereas postadaptation generalization reflects the final weight vectors after adaptation is completed.

We assumed that learning proceeds through modification of the weight vectors and not through modification of the neurons' preferred directions $\left\{\theta_{i}\right\}$. Alternatively, the gradient descent learning rule can just as easily be applied to changes in preferred direction

$$
\Delta \theta_{i}=-\eta \frac{\partial \varepsilon}{\partial \theta_{i}}=\eta \frac{\partial g\left(\theta-\theta_{i}\right)}{\partial \theta_{i}}\left(\vec{d}_{i} \cdot \Delta \vec{r}\right)
$$

and the weight vectors would remain constant. However, if we assume a symmetric tuning function as found in most visual and motor regions, this learning rule will lead to an asymmetric pattern of trial-by-trial generalization. This is because the derivative of an even (symmetric) function is an odd (anti-symmetric) function. Experimentally, patterns of both trial-by-trial and postadaptation generalization are symmetric with respect to a learned target direction. Thus a learning rule based on changing preferred directions would not reproduce the experimental data.

SIMULATION DETAILS. All the simulations and the data analyses were performed using MATLAB (The MathWorks, Natick, MA) on a Dell Precision Workstation 670 (2 Xeon Irwindale 3.6 GHz) running the Linux operating system and on a Dell Precision Workstation PSW490 (Xeon $2.0 \mathrm{GHz}$ ) running the Windows operation system. In the following simulations, unless otherwise stated we used a Gaussian function of width $\sigma$

$$
g(\theta)=\frac{1}{\sqrt{2 \pi \sigma^{2}}} \exp \left(-\frac{\theta^{2}}{2 \sigma^{2}}\right)
$$

initialized the weight vectors, and trained the model using the gradient learning rule as described in the preceding text. Note that the Gaussian functional form was chosen primarily for mathematical simplicity in describing a unimodal function. We fixed $\sigma=23^{\circ}$ and $\eta=4.0$. The number of units $N$ was taken to be 15 , and we confirmed that increasing $N$ did not dramatically change the following results if the learning rate constant was adjusted accordingly as shown in the previous paragraph.

The learning rate was chosen to match the experimentally observed learning speed. The normalization factor in front of the tuning function was introduced to keep the sum of population activity at the same level regardless of its tuning width. Note that the two parameters (the number of units $N$ and the learning rate $\eta$ ) are irrelevant for both postadaptation and trial-by-trial generalization patterns. Because $\eta$ determines the learning speed but does not affect the final remapping, it does not affect postadaptation generalization, which by definition is measured following learning. Also as understood from Eq. 13, the learning rate scales in all directions uniformly and therefore does not change the trial-by-trial generalization pattern. Thus the only parameter relevant for determining the shape of generalization patterns is the tuning width, $\sigma$. The value of the tuning width in the simulations $\left(23^{\circ}\right)$ was constrained by the experimentally observed generalization functions. The trial-by-trial analysis, on the one hand, required that trial-by-trial generalization fall close to zero for $\geq 45^{\circ}$ separation, imposing an upper limit on the tuning width. The postadaptation analysis, on the other hand, showed some transfer at $22.5^{\circ}$, suggesting a small but nonzero value for the tuning width.

\section{RES U L T S}

\section{Broad tuning functions cannot explain local postadaptation generalization of single-target rotation learning}

Using the population coding model, we could test a wide range of tuning functions that have been found in different brain regions to see what they predict for visuomotor rotation generalization (Andersen et al. 1985; Coltz et al. 1999; Georgopoulos et al. 1982).

We computed the postadaptation generalization patterns after single target training based on three tuning functions: narrow Gaussian tuning $\left(E q .16, \sigma=23^{\circ}\right)$, cosine tuning $[\cos (\theta)]$, found in primary motor cortex (Georgopoulos et al. 1982) and area 5 in the parietal cortex (Kalaska et al. 1990), and the bimodal twoGaussian tuning $\left\{\exp \left(-\theta^{2} / 2 \sigma^{2}\right)+\exp \left[-(\theta-180)^{2} / 2 \sigma^{2}\right] / K, \sigma=\right.$ $\left.34^{\circ}, K=1.7\right\}$ found in the cerebellum (Coltz et al. 1999). We then compared the generalization predicted by the three different tuning functions. In Fig. $1 A$, the cosine and the twoGaussian directional tuning led to much wider generalization functions. The largest discrepancy between the different tuning functions was apparent at $180^{\circ}$ (direction opposite to the trained target). In the case of two-Gaussian tuning, the population activity for one target direction and that for the opposite direction overlapped positively, so the error vector experienced at one target direction was transferred with the same sign to the $180^{\circ}$ direction according to Eq. 13 (see Fig. $1 B$ ). This positive vector transfer interferes with rotation learning, however, because the changes in movement direction required for opposite target directions should be of opposite sign. In the case of cosine tuning, population activities for opposite target directions overlap negatively, and thus an error vector at one direction was transferred to the opposite direction with the sign inverted. Thus the broad tuning functions that have been found experimentally cannot explain narrow generalization and the absence of generalization to $180^{\circ}$ for visuomotor rotation can only be explained by narrow unimodal tuning. 
A

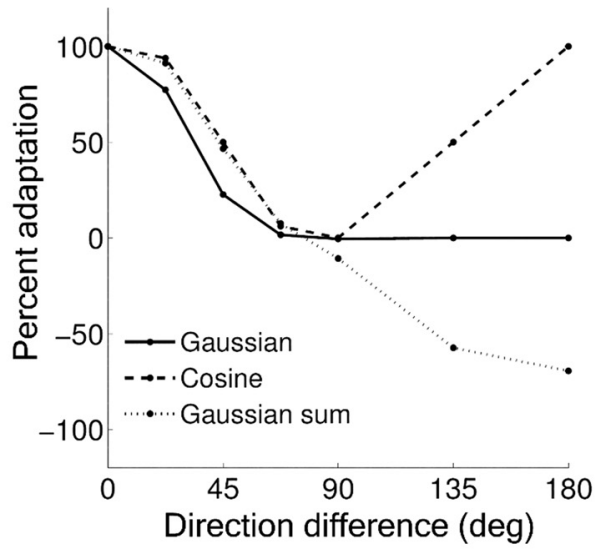

B

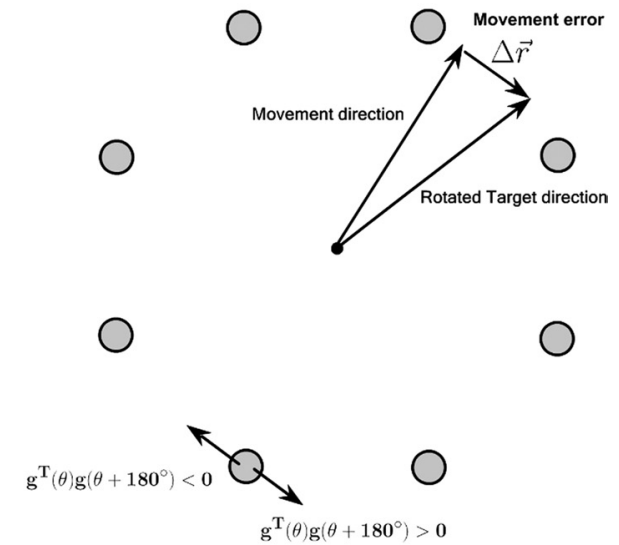

FIG. 1. Relationship between tuning shape and postadaptation generalization function shape. A: extent of postadaptation generalization computed with 3

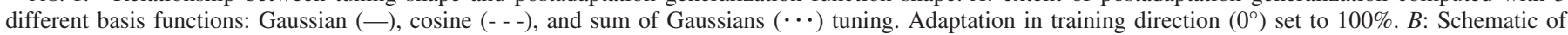

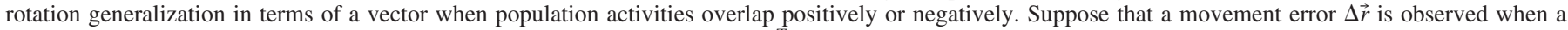

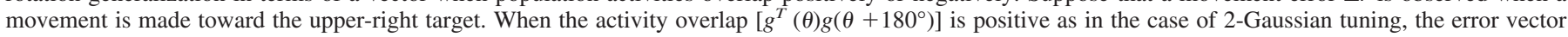

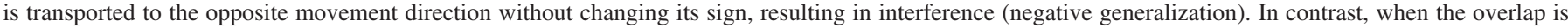

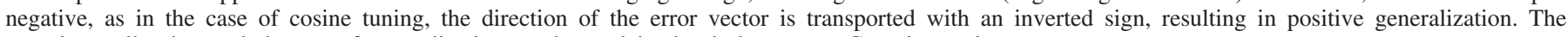
experimentally observed absence of generalization can be explained only by narrow Gaussian tuning curves.

In the cerebellum, directional tuning functions are not homogeneous but can take several forms (Coltz et al. 1999). One possibility is that a mixed representation of cosine and Gaussian-sum tuning could explain null generalization beyond $90^{\circ}$ as their respective positive and negative generalization would then cancel each other out. To address this issue, we constructed a population coding model using a mixture of cosine and Gaussian-sum tuning as

$$
\vec{r}=\sum_{i=1}^{N_{\text {cos }}} \vec{d}_{i}^{\text {cos }} g_{i}^{\text {cos }}(\theta)+\sum_{i=1}^{N_{\text {Gauss-sum }}} \vec{d}_{i}^{\text {Gauss-sum }} g_{i}^{\text {Gauss-sum }}(\theta)
$$

and varied the proportion $N_{\text {cos }} / N_{\text {Gauss-sum }}$ from 0 to $100 \%$ in step of $20 \%$ (Fig. 2A). A numerical simulation confirmed that a population-coding model composed of $20 \%$ cosine and $80 \%$ two-Gaussian tuning indeed showed null generalization beyond $90^{\circ}$, consistent with experimental results (Krakauer et al. 2000). This mixture proportion is, however, not found in the cerebellum where only a minority of cells $(12-18 \%)$ showed bimodal tuning (Coltz et al. 1999), and even in this case, there was considerable generalization at $45^{\circ}$ separation $(\sim 50 \%)$, which is wider than the generalization observed for rotation adaptation.
Population vectors are usually computed as sums using the differences in firing rates from their mean values rather than the raw firing rates (see, e.g., Eq. 2 of Georgopoulos et al. 1988), so offsets in cosine tuning are not important. However, the degree of generalization depends on the overlap of two population activities, which does depend on the offset values, so it is of interest to examine how offsets affect the degree of postadaptation generalization. We performed a numerical simulation using cosine tuning with an offset term

$$
\vec{r}=\sum_{i=1}^{N} \vec{d}_{i}\left[g_{i}^{\cos }(\theta)+g_{0}\right]
$$

where the offset was varied from 0 to 1.2 in steps of 0.3 (Fig. $2 B$ ). Similarly in the mixed population in the preceding text, the population vector for $g_{0}=0.6$ and an offset produced almost null generalization beyond $90^{\circ}$ but showed considerable generalization at $45^{\circ}$, inconsistent with experimental findings. These simulation results provide further support that only narrow unimodal Gaussian tuning can explain the narrow shape of postadaptation generalization found experimentally for rotation learning.
A

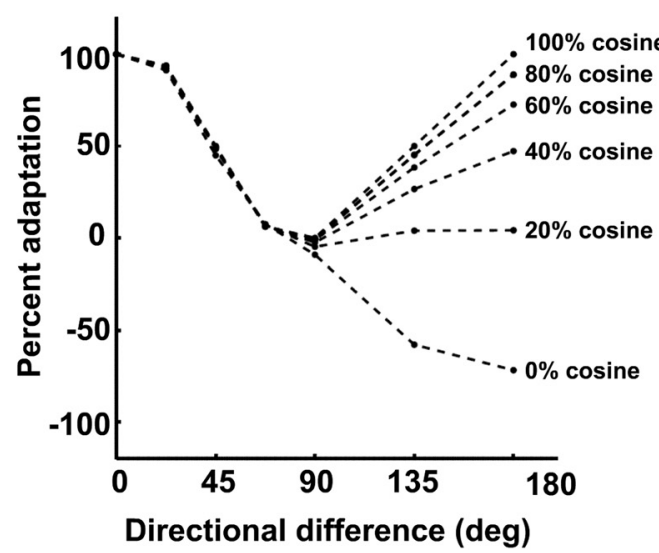

B

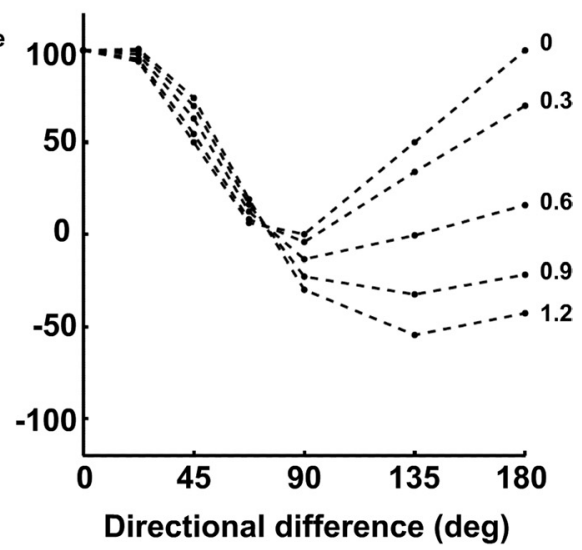

FIG. 2. Effects on postadaptation generalization shapes when a mixed population of cosine and Gaussian-sum tuning functions was considered $(A)$ and when an offset term was added to the cosine tuning function $(B)$. The numbers accompanied by the curves denote the percentages of cosine tuned units in the mixed population in $A$ and the offset values in $B$, respectively. 


\section{Population-coding model with narrow Gaussian tuning predicts adaptation rate and postadaptation generalization in multi-target rotation learning}

Assuming that rotation adaptation might depend on neurons with narrow tuning functions, we asked whether narrow tuning widths could explain two previous experimental results in multi-target rotation learning (Krakauer et al. 2000. The first finding was that the adaptation rate decreased gradually as the number of training target increased and that the adaptation rate divided by the number of visits to a target was found to be almost constant. This implies that adaptation at one target was independent of visits to the other targets, which provides an upper limit on the tuning width. The second finding was that the degree of postadaptation generalization gradually increased as the number of training targets increased.

AVERAGE LEARNING RATE. Can the population-coding model explain the exponential decay of directional error during adaptation? We first simulated the eight-target experiment with a randomized target order. We generated a trial-by-trial vectorial error time series with the population-coding model assuming a narrow Gaussian tuning function of width $23^{\circ}$, converted the vectorial errors into scalar angular errors and fitted them with an exponential function. To simulate planning noise (Gordon et al. 1994: van Beers et al. 2004), we added independent Gaussian noise, with a SD of $5 \%$ of the hand-movement amplitude, to the output vector, $\vec{r}$. Gray circles for both the experimental and modeling data represent errors observed at each trial in the trial-by-trial error sequence. Figure $3 B$ demonstrates that the model matched the trend in the eight-target experiment $(A)$, and the noise in the movements.

We then examined how the number of training targets $(1,2$, 4 , or 8 ) influenced the adaptation rate. In agreement with the experimental findings (Fig. 4A), the model reproduced the gradual decrease in the learning rate as the number of target increased $(B)$. The learning speeds divided by number of targets were almost constant. The constant learning rate reproduced in the model can be explained by narrow tuning width: training in one direction was almost independent of learning in other directions. Therefore the activity overlap between adjacent targets should be small, and the observed independent learning interaction across targets separated by $45^{\circ}$ imposed an upper limit on the tuning function width.

POSTADAPTATION GENERALIZATION. In previous work, we found that a $30^{\circ}$ rotation learned to a single target did not generalize beyond $90^{\circ}$ (Krakauer et al. 2000). However, postadaptation generalization increased as the number of training directions sampled increased (Fig. 5A). To confirm that the population-coding model could reproduce these generalization patterns, we trained the model fully on the $30^{\circ}$ counterclockwise rotation for one, two, four, and eight targets and then, after training reached asymptote, we tested the extent to which the model generalized to other untrained directions. Figure $5 B$ shows that the model successfully reproduced the experimental generalization pattern although there was a small discrepancy at $90^{\circ}$ for two-target learning. Whereas the postadaptation generalization function computed from the experimental data shows small positive values at $90^{\circ}$ separation and beyond, the function computed from the population-coding model takes almost zero values. The tuning function in the model (Eq. 16) did not include a constant term, which would have contributed uniformly to generalization for all target directions. Our main interest in this study was to investigate the width of generalization but not the value itself, so for simplicity we did not introduce a constant term in the tuning function. The shape of the postadaptation generalization functions were all nearly symmetric with respect to trained target directions, which supported the hypothesis that the weight vectors and not the preferred unit directions were subject to modification during visuomotor adaptation.

\section{Narrow directional tuning predicts trial-by-trial generalization}

We next investigated whether the population-coding model with narrow Gaussian tuning could also explain trial-by-trial generalization of rotation as shown previously for force-field adaptation (Donchin et al. 2003; Thoroughman and Shadmehr 2000). In the population-coding model, a directional error experienced at the $k$ th trial led to an update of the weight vectors, as shown in $E q .12$, and these updated weight vectors in turn influenced the movement direction at the $(k+1)$ th trial, as in Eq. 13. Therefore the model described trial-by-trial generalization as a direct consequence of changes in the weight vectors. In principle, though, the same results could have been obtained by changing the preferred target directions, $\left\{\theta_{i}\right\}$, but this would not have reproduced the nearly symmetric patterns of generalization (see the paragraph containing Eq. 15 in METHODS).

Figure $6 A$ shows the trial-by-trial errors from a representative single run from one participant (solid black line). The single-state model was fitted by adjusting $B$ and $\Phi_{1}$ and accounted well for the trial-by-trial errors (dashed gray line). The $R^{2}$ value of this participant was 0.806 and that averaged across the 12 participants was 0.715 . To confirm that trial-by-
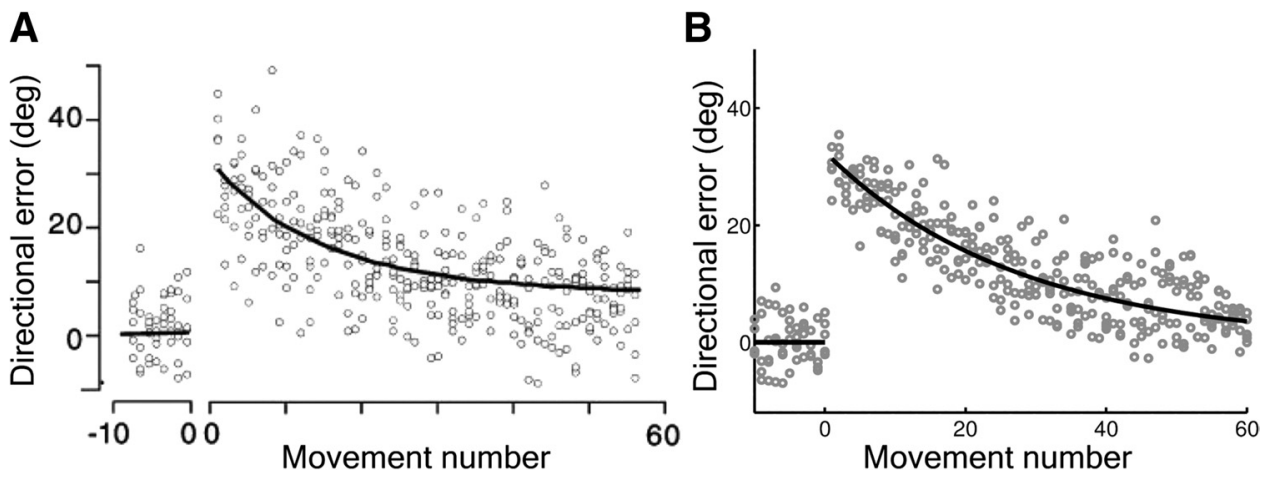

FIG. 3. Rotation learning. Trial-by-trial errors (open gray circles) and fitted learning curves (thick black lines) for eight-target training. A: experimental data taken from Krakauer et al. (2000), with permission; $B$ : simulated errors derived from the population-coding model. Trials -10 to 0 are at baseline (visual feedback, no rotation). In the simulation, 5 independent runs were computed, and the average learning curve is shown. 
A

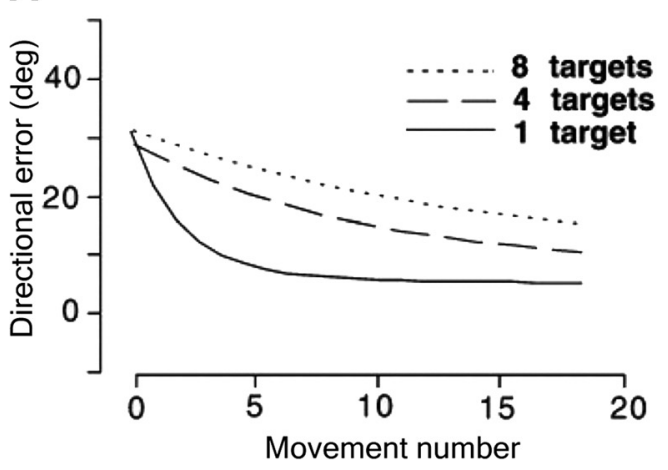

B

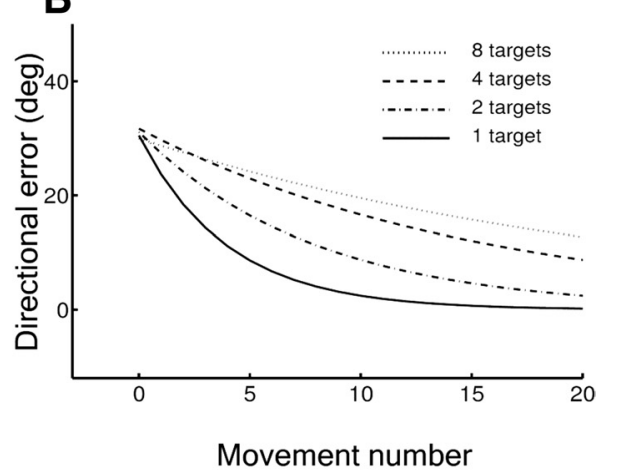

FIG. 4. Fitted learning curves for single-, 4- and 8-target rotation training from Krakauer et al. (2000]) (A), with permission, and simulated with the population-coding model $(B)$. trial generalization was direction dependent, we compared these results with a model using a single exponential function, which can be obtained from the state-space model assuming uniform generalization among the targets. Fitting the same initial 70 trials using a single exponential yielded 0.301 of the $R^{2}$ value; an $F$-test indicated that the fit with state-space model was significantly better than with the single-exponential for all participants, including the participant with the worst statespace fit $[F(14,70)=2.63$ at $P<0.01$ level]. Thus the success of the state-state model in capturing the characteristics of trial-by-trial adaptation in the initial phase of rotation learning was not just a consequence of having an exponential in the learning curves. Three additional participant cases are included in the supplementary materials.

We then generated artificial trial-by-trial time series assuming the same narrow Gaussian width used for the simulation of postgeneralization adaptation (solid black line in Fig. 6C). We fitted the time series with the state-state model (dashed gray line in Fig. 6C) to compute the generalization function. To simulate the noisy components that were observed experimentally, we added two-dimensional Gaussian noise vectors to the output vector $\vec{r}$ of the model, whose SD was $5 \%$ of $|\vec{r}|$. The model in Fig. $6 D$ exhibited a narrow pattern that is localized to no more than $45^{\circ}$, in close agreement with the experimentally derived generalization function $(B)$.

It is worth noting that the localized trial-by-trial generalization pattern that we found both experimentally and with the population-coding model is in sharp contrast to the much broader generalization pattern found for force-field adaptation (Donchin et al. 2003; Thoroughman and Shadmehr 2000). This difference supports the idea that kinematic adaptation and dynamic adaptation are distinct processes mediated by separate neuronal populations (see DISCUSSION).

Change in weight vectors after rotation adaptation explains single-unit recording results in primary motor cortex

In recent single-unit recording studies (Paz and Vaadia 2004; Paz et al. 2003), changes in the preferred direction of neurons in primary motor cortex (M1) were investigated as monkeys adapted to rotations of various magnitudes to a single target. It was found that neuronal activity was enhanced in neurons whose preferred directions were close to actual handmovement direction, regardless of the rotation angle learned. These findings led to the proposal that M1 was a locus for visuomotor rotation learning.

To interpret this physiological finding in the light of our computational model, we examined how weight vectors changed after simulating single-target training with $-90,-45$, 45 , or $90^{\circ}$ rotations. Using the same narrow Gaussian tuning function, the population-coding model learned the rotations by modifying the weight vectors $\left\{d_{i}\right\}$ between visually-selective and motor areas. The pattern of changes in the vectors after learning provides information on how the remapping was encoded in the model.

There were two findings for the postadaptation weight vectors (Fig. 7). First, an excess of motor output vectors clustered (within $\pm 30^{\circ}$ ) around the actual hand-movement direction. Second, significantly fewer motor vectors remained in visual target directions. Thus regardless of the magnitude of the imposed rotation, the change in motor output vectors showed a consistent behavior: the weight vectors that originally pointed
A

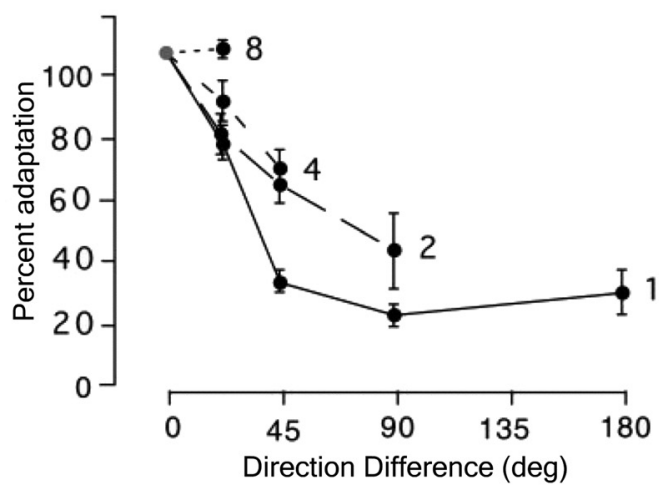

B

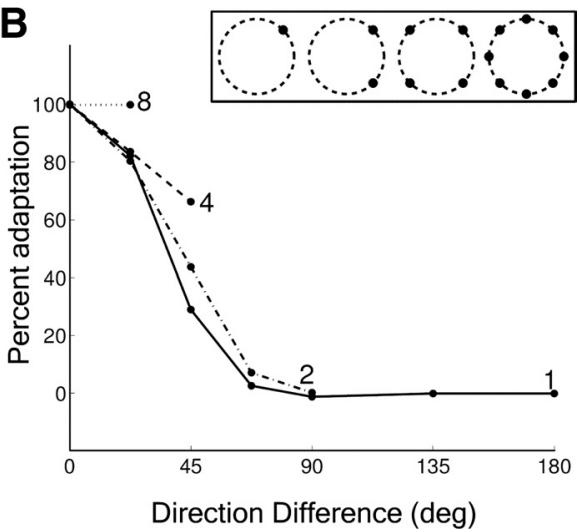

FIG. 5. Degrees of postadaptation generalization after training to $1,2,4$ or 8 target directions, taken from Krakauer et al. (2000) (A) with permission, and computed with the population-coding model $(B)$. In $B$, training directions were shown in the inset. Data collapsed for clockwise and counter-clockwise testing directions. Training direction $=0^{\circ}$. 
A

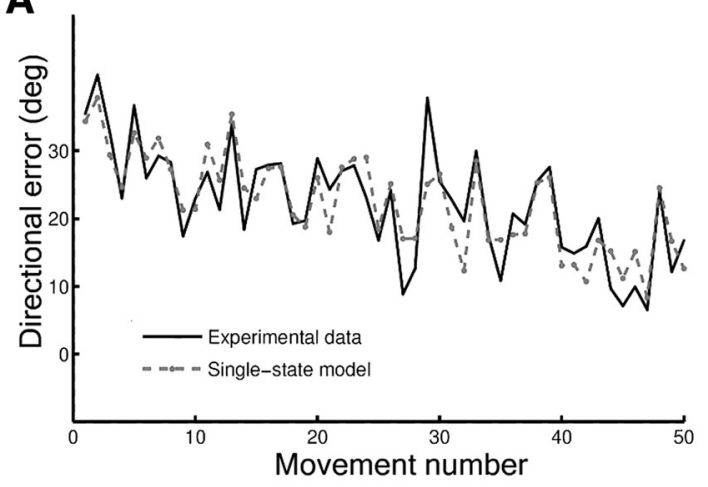

C

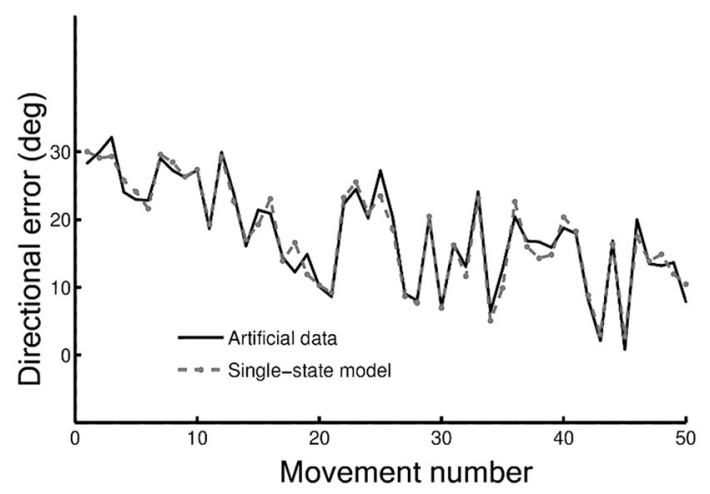

B
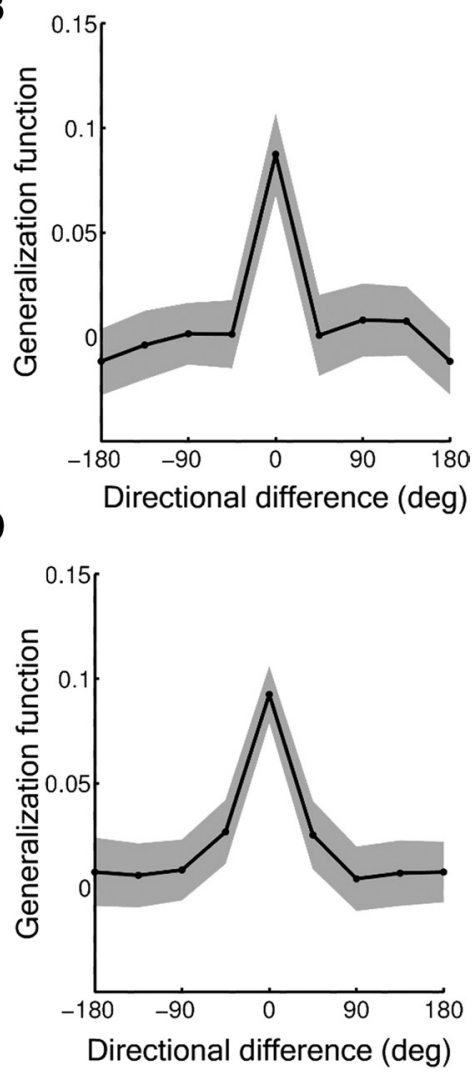

FIG. 6. A: representative trial-by-trial errors observed in a single experiment from 1 participant; $B$ : the corresponding generalization function computed from the experimental data of all 12 participants using a singlestate state-space model. $C$ : single-run artificial data generated by the population-coding model for which the tuning width was used in the postadaptation generalization and the corresponding generalization function $(D)$. In $A$ and $C$, the trial-by-trial errors were fitted by the single-state state-space model to demonstrate how well the single-state model explains the error sequences. Shaded areas accompanying the generalization functions indicate $95 \%$ confidence intervals. toward the target location before rotation learning moved toward the direction of the actual hand-movement.

These findings provide an alternative interpretation for the single-unit recordings reported by Paz and Vaadia (Paz and Vaadia 2004; Paz et al. 2003). Suppose that the narrowly tuned computational units in our model represent neurons that are selectively tuned to visually perceived movement directions and that they project to downstream neurons that are selective for particular hand-movement directions. Then the weight vector $\left\{\vec{d}_{i}\right\}$ represents the connection from an area that plans movement in visual coordinates to a downstream area that controls the actual limb movement direction. Initially, before rotation learning, the mapping from visual to motor coordinates is distributed uniformly across directions: visually perceived and executed movement directions are same. During learning, those neurons whose preferred directions are congruent with the desired trajectory of upstream visually selective neurons show progressive activity enhancement as the result of an increase in the synaptic weights between the respective upstream visual and downstream motor neurons.

Note that this change in the weight vectors occurs only in the vicinity of the actual hand-movement direction because upstream tuning is narrow and unimodal, consistent with the physiological result that the activity enhancement was observed only in the selective population of M1 neurons tuned to the actual hand-movement direction. Therefore our model proposes that the newly learned visuomotor remapping is stored in the connections between upstream visual areas and downstream motor areas, and that the activity enhancement reported in M1 (Paz and Vaadia 2004) is a consequence of these changes in synaptic strengths from inputs.
Population-coding model predicts hyper-adaptation within the generalization width

The population-coding model makes the testable prediction the visuomotor remapping can be rotated beyond that imposed in the experiment. The model is first adapted fully to a single target and then readapted fully to an adjacent target within the generalization width. The model predicts that the remapping of the first target on the completion of second adaptation is "hyperadapted," i.e., rotated more than the visual rotation due to the transfer of learning to the second target. A numerical simulation using the population-coding model with narrow directional tuning predicted $\sim 20 \%$ hyperadaptation $\left(36^{\circ}\right.$ rotated remapping when $30^{\circ}$ visual rotation was imposed) if followed by adaptation to a second target that is separated by $22.5^{\circ}$.

\section{I S C U S S I O N}

A population coding model based on a single population of neurons with narrow tuning accounted for many experimental aspects of visuomotor rotation learning. It explained the observed constant learning speed per target, postadaptation generalization and trial-by-trial generalization. The population coding model also provided insights into possible anatomical sites and physiological mechanisms for rotation adaptation.

The psychophysical findings implied narrow directional tuning for the model neurons and ruled out cosine or sum-ofGaussians tuning curves. Our model also predicted that it is the output connections (weight vectors) and not the preferred tuning directions that are subject to modification by visual errors; that is, the remapping was encoded as changes in 
A

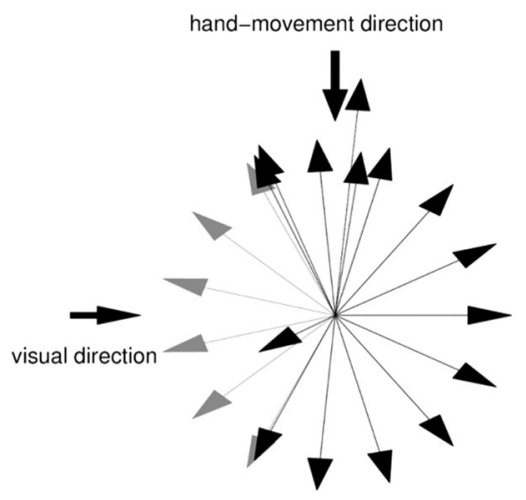

C

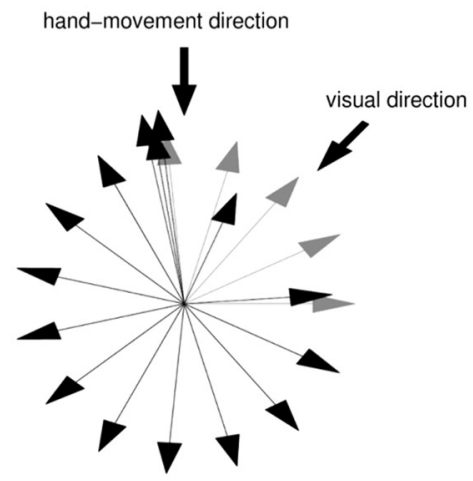

B

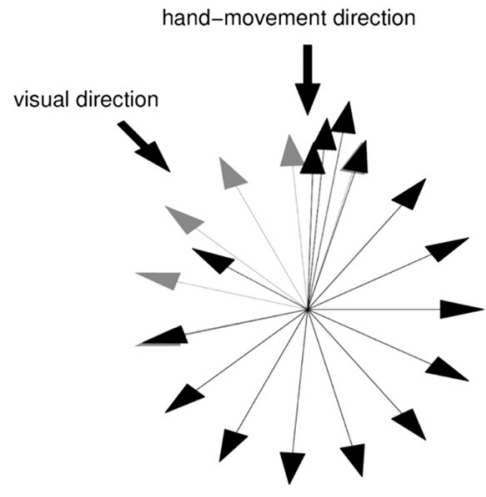

D

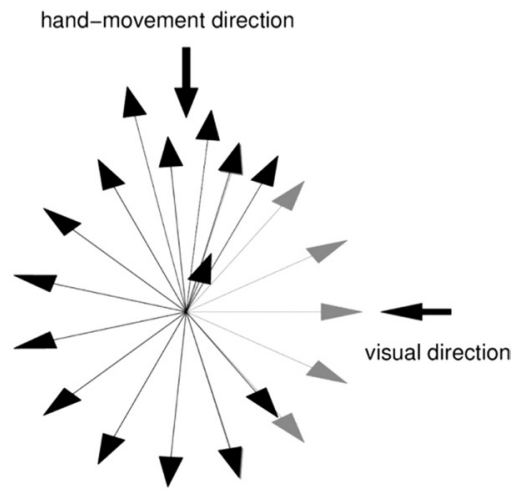

FIG. 7. Changes in the weight vectors after training the model in a single-target experiment under $-90(A),-45(B),+45(C)$, or $+90^{\circ}(D)$ rotation. The gray arrows represent the weight vectors prior to rotation learning, which are uniformly distributed over all directions, and the black arrows represent the weight vectors subsequent to the rotation learning. For illustration, the executed handmovement directions are fixed at $90^{\circ}$ (away from the body), and the corresponding visual target directions are indicated by short black arrows. synaptic weights between sensory and motor areas. The enhancement of activity in selective populations of directionally tuned cells in M1 that has been observed after rotation adaptation (Paz and Vaadia 2004) is consistent with changing the strengths of connections from upstream posterior parietal areas to downstream motor areas.

\section{Comparisons with previous computational models}

Our population-coding model is consistent with previous computational models in the sense that use of sensory information can vary depending on the task, the context, or the effector. Based on models of functional approximation using basis functions and gain fields, a nonlinear transformation in any coordinate system or reference frame can in principle be calculated from the outputs of posterior parietal cortex (Pouget and Sejnowski 1997). The sensory representation in these models is invariant with respect to how it is used downstream, consistent with our assumption that the output weight vectors change rather than the tuning functions. Thus our model makes the testable physiological prediction that the tuning functions responsible for visuomotor transformation in posterior parietal cortex should not change their shapes or preferred directions during rotation learning, whereas there should be the changes in motor areas as described by Paz and Vaadia (Paz et al. 2003) and illustrated schematically in Fig. 7.

\section{Shapes of the tuning curves used in the population-coding model}

The population-coding model assumed that neurons with a preferred direction in visual space congruent with the desired target direction would, over the course of adaptation, increase the strength of their connections with neurons in motor/premotor cortex with a preferred direction in the required rotated hand-movement direction. The model successfully explained the dependence of adaptation rate on training target number and the narrow generalization functions obtained using both postadaptation and the state-space modeling approaches.

Other forms of neural tuning that were tested in the model, such as the broad cosine tuning found in M1 (Georgopoulos et al. 1982) or the broader tuning found in cerebellum (Coltz et al. 1999), produced generalization functions inconsistent with our empirical results. In contrast, the generalization functions for force field learning may be compatible with the broader tuning found in the cerebellum (Donchin et al. 2003). Thus the necessity for narrow Gaussian directional tuning in the model supports different neural correlates for rotation adaptation and adaptation to novel dynamics (Flanagan et al. 1999; Krakauer et al. 1999) and constrains the possible anatomical candidates for processing of directional error during rotation adaptation.

\section{Postadaptation generalization and trial-by-trial generalization may be related}

Generalization of sensorimotor adaptation has traditionally been assessed through a postadaptational block-design experiment in which the untrained workspace is tested after remapping the fully trained workspace. In contrast, trial-by-trial adaptation assesses how an error on one trial influences the performance on subsequent trials during the time course of adaptation. These two types of generalization need not to be same. The finding that trial-by-trial generalization and postad- 
aptational generalization were identical for visuomotor rotation learning is in sharp contrast with force-field adaptation, where there was a significant difference between the two types of generalization: Motor adaptation evaluated postadaptationally did not transfer beyond $90^{\circ}$ (Mattar and Ostry 2007) whereas trial-by-trial generalization showed much broader patterns (Donchin et al. 2003; Huang and Shadmehr 2007; Thoroughman and Shadmehr 2000; Thoroughman and Taylor 2005).

The two types of generalization may originate from separate but interacting processes of motor adaptation. In a recent, innovative multi-state model, several, concurrent processes in motor adaptation are explicitly represented - a fast process with a fast learning rate but weak retention and a slow process with a slow learning rate but strong retention (Smith et al. 2006). We show elsewhere, through analysis and numerical simulations, that trial-by-trial generalization predominantly relates to the fast process in the two-state model, whereas postadaptational generalization predominantly relates to the slow process.

\section{Parietal cortex as the site for visuomotor rotation learning}

One candidate area for rotation learning is the posterior parietal cortex. Several pieces of evidence support this conjecture. First, imaging studies have found activation of the parietal cortex when participants adapt to rotation (Clower et al. 1996; Diedrichsen et al. 2005; Inoue et al. 1997, 2000; Krakauer et al. 2004). Second, studies of prism adaptation, a related visuomotor transformation, suggest that the transformation occurs in the parietal reach region, which may code movement goals in visual coordinates (Fernandez-Ruiz et al. 2007; Gail and Andersen 2006). In support of this possibility, a patient with bilateral posterior parietal cortex damage was unable to adapt to a prism (Newport et al. 2006). Third, the tuning width we used for the model was within the range found for the receptive fields of visually selective cells in area $7 \mathrm{a}$ of posterior parietal cortex (Andersen et al. 1985). Although the connections between parietal cortex and motor cortex are likely sites for synaptic plasticity, it is also possible that remapping occurs within motor cortex at synapses between one subgroup of neurons reflecting narrowly tuned visual inputs from parietal areas and another subgroup sending broadly tuned movement outputs. There may be multiple sites within the cortex that support motor remapping, all of which are consistent with the assumptions and predictions of the computational models studied here.

The model presented here suggests that a change in the weights of connections between posterior parietal cortex and motor areas is the locus for adaptation to rotation. This conclusion seems to contradict conclusions from studies that have suggested instead that the locus of adaptation is in M1 (Paz and Vaadia 2004; Wise et al. 1998), premotor cortex (Wise et al. 1998), or the cerebellum (Tseng et al. 2007). A recent single-unit study proposed that the neuronal correlates of rotation learning are to be found in primary motor cortex (M1) based on the finding of activity enhancement in neurons the preferred direction of which is approximately congruent with the postadaptation hand-movement direction (Paz et al. 2003). We would argue, however, that this finding cannot explain the neuronal mechanism that underlies visuomotor rotation because the degree of activity enhancement was independent of the magnitude of the rotation. What has to be learned is not the neural encoding of the required hand-movement direction postadaptation but the rotated mapping between the visually perceived trajectory and hand-movement direction. Our model was able to reproduce and thereby provide an alternative explanation for these findings in motor cortex - the activity enhancement in motor cortex reflects the modification in the output vectors upstream to motor cortex: i.e., the rotated mapping itself is stored in the weight vectors representing the connections from posterior parietal cortex to motor cortex (Strick and Kim 1978; Zarzecki et al. 1978).

\section{Role of the cerebellum in visuomotor mapping}

A recent study showed impairment in rotation adaptation in patients with cerebellar disease (Tseng et al. 2007), consistent with previous studies reporting impaired prism adaptation in patients with cerebellar lesions (Martin et al. 1996). The cerebellum has been proposed as a site for forward models (Miall et al. 1993), from which it is possible to compute a prediction error between the expected and the observed trajectory (Wolpert and Miall 1996). In the case of visuomotor rotation learning, the prediction error between the expected movement direction predicted by the forward model $(\vec{r})$ and the observed cursor direction $\left(\vec{r}_{c}\right)$ was used to compute the rotation matrix in Eq. 4. In a recent study, we showed that rotation adaptation likely occurs via a forward model (Mazzoni and Krakauer 2006). Thus we would suggest that the cerebellum provides the prediction error of the movement vector that drives the change in synaptic weights in posterior parietal cortex. This is supported by studies showing that posterior parietal cortex is a target for outputs from the cerebellum (Clower et al. 2001); indeed the authors of this study concluded: "we suggest that the cerebellar projection to posterior parietal cortex may provide signals that contribute to (or initiate) the sensory recalibration that occurs during the adaptive process."

\section{Conclusions}

The overall goal of this study was to use modeling approaches to bridge experimental results with their underlying neuroanatomical substrate (Shadmehr and Krakauer 2008). A population coding model with a single population of narrow Gaussian-tuned neurons was able to reproduce the experimentally derived generalization functions and learning curves and provided an alternative interpretation for the postadaptation activity enhancement that has been reported in selective M1 neurons after rotation adaptation (Paz and Vaadia 2004; Paz et al. 2003). We propose the following sequence of events: first, the cerebellum computes a prediction error for the movement vector via a forward model. Second, this prediction error is projected to neurons in posterior parietal cortex that have narrow directional tuning. Third, the synaptic weights between these neurons in posterior parietal cortex and motor cortical areas are modified to reduce the prediction error, which leads to a remapping between the reach trajectory in visual space and movement direction in hand space. This remapping would represent a new inverse model or controller in visual space, which suggests that in our framework a forward model trains an inverse model. Finally, these changes in the weights are 
reflected in increased activity in neurons in motor and/or premotor cortex whose preferred direction in hand space matches the required visual trajectory.

\section{A C K N O W L E D G M EN T S}

We thank J. Liang for assistance in conducting the psychophysical experiments, and P. Strick for discussions regarding the anatomy underlying the model. H. Tanaka thanks R. Natarajan and R. Shadmehr for stimulating discussions during the Okinawa Computational Neuroscience Course 2005 where a part of this work was initiated.

\section{G R A N T S}

This research was supported by the Howard Hughes Medical Institute (T. J. Sejnowski) and NINDS R01 052804 (J. W. Krakauer).

\section{REFERENCES}

Andersen RA, Essick GK, Siegel RM. Encoding of spatial location by posterior parietal neurons. Science 230: 456-458, 1985.

Bishop CM. Neural Networks for Pattern Recognition. Oxford, UK: Oxford Univ. Press, 1995

Cheng S, Sabes PN. Modeling sensorimotor learning with linear dynamical systems. Neural Comput 18: 760-793, 2006.

Clower D, Hoffman J, Votaw J, Faber T, Woods R. Role of posterior parietal cortex in the recalibration of visually guided reaching. Nature 383: 618-621, 1996.

Clower DM, West RA, Lynch JC, Strick PL. The inferior parietal lobule is the target of output from the superior colliculus, hippocampus, and cerebellum. J Neurosci 21: 6283-6291, 2001.

Coltz JD, Johnson MT, Ebner TJ. Cerebellar Purkinje cell simple spike discharge encodes movement velocity in primates during visuomotor arm tracking. J Neurosci 19: 1782-1803, 1999.

Della-Magglore V, McIntosh AR. Time course of changes in brain activity and functional connectivity associated with long-term adaptation to a rotational transformation. J Neurophysiol 93: 2254-2262, 2005.

Diedrichsen J, Hashambhoy Y, Rane T, Shadmehr R. Neural correlates of reach errors. J Neurosci 25: 9919-9931, 2005.

Donchin O, Francis JT, Shadmehr R. Quantifying generalization from trial-by-trial behavior of adaptive systems that learn with basis functions: theory and experiments in human motor control. J Neurosci 23: 9032 $9045,2003$.

Efron B. The Jackknife, the Bootstrap and Other Resampling Plans. Philadelphia: Society for Industrial and Applied Mathematics, 1982.

Fernandez-Ruiz J, Goltz HC, Desouza JF, Vilis T, Crawford JD. Human parietal "reach region" primarily encodes intrinsic visual direction, not extrinsic movement direction, in a visual-motor dissociation task. Cereb Cortex 17: 2283-2292, 2007.

Flanagan JR, Nakano E, Imamizu H, Osu R, Yoshioka T, Kawato M. Composition and decomposition of internal models in motor learning under altered kinematic and dynamic environments. J Neurosci 19: 1-5, 1999.

Gail A, Andersen RA. Neural dynamics in monkey parietal reach region reflect context-specific sensorimotor transformations. J Neurosci 26: $9376-$ 9384, 2006.

Georgopoulos AP, Kalaska JF, Caminiti R, Massey JT. On the relations between the direction of two-dimensional arm movements and cell discharge in primate motor cortex. J Neurosci 2: 1527-1537, 1982.

Georgopoulos AP, Kettner RE, Schwartz AB. Primate motor cortex and free arm movements to visual targets in three-dimensional space. II. Coding of the direction of movement by a neuronal population. J Neurosci 8: 2928-37, 1988.

Ghahramani Z, Wolpert DM, Jordan MI. Generalization to local remappings of the visuomotor coordinate transformation. J Neurosci 16: 70857096, 1996.

Goodwin GC, Sin KS. Adaptive Filtering: Prediction and Control. Englewood Cliffs, NJ: Prentice-Hall, 1984.

Gordon J, Ghilardi MF, Ghez C. Accuracy of planar reaching movements. I. Independence of direction and extent variability. Exp Brain Res 99: 97-111, 1994.

Hadipour-Niktarash A, Lee CK, Desmond JE, Shadmehr R. Impairment of retention but not acquisition of a visuomotor skill through time-dependent disruption of primary motor cortex. J Neurosci 27: 13413-13419, 2007.

Huang VS, Shadmehr R. Evolution of motor memory during the seconds after observation of motor error. J Neurophysiol 97: 3976-3985, 2007.
Imamizu H, Uno Y, Kawato M. Internal representations of the motor apparatus: implications from generalization in visuomotor learning. $J$ Exp Psychol Hum Percept Perform 21: 1174-1198, 1995.

Inoue K, Kawashima R, Satoh K, Kinomura S, Goto R, Sugiura M, Ito M, Fukuda H. Activity in the parietal area during visuomotor learning with optical rotation. Neuroreport 8: 3979-3983, 1997.

Inoue K, Kawashima R, Satoh K, Kinomura S, Sugiura M, Goto R, Ito M, Fukuda H. A PET study of visuomotor learning under optical rotation. Neuroimage 11: 505-516, 2000.

Kagerer FA, Contreras-Vidal JL, Stelmach GE. Adaptation to gradual as compared with sudden visuo-motor distortions. Exp Brain Res 115: $557-$ $561,1997$.

Kalaska JF, Cohen DA, Prud'homme M, Hyde ML. Parietal area 5 neuronal activity encodes movement kinematics, not movement dynamics. Exp Brain Res 80: 351-364, 1990.

Krakauer JW, Ghilardi MF, Ghez C. Independent learning of internal models for kinematic and dynamic control of reaching. Nat Neurosci 2: 1026-1031, 1999.

Krakauer JW, Ghilardi MF, Mentis M, Barnes A, Veytsman M, Eidelberg D, Ghez C. Differential cortical and subcortical activations in learning rotations and gains for reaching: a PET study. J Neurophysiol 91: 924-933, 2004.

Krakauer JW, Pine ZM, Ghilardi MF, Ghez C. Learning of visuomotor transformations for vectorial planning of reaching trajectories. $J$ Neurosci 20: 8916-8924, 2000

Malfait N, Shiller DM, Ostry DJ. Transfer of motor learning across arm configurations. J Neurosci 22: 9656-9660, 2002.

Martin TA, Keating JG, Goodkin HP, Bastian AJ, Thach WT. Throwing while looking through prisms. I. Focal olivocerebellar lesions impair adaptation. Brain 119: 1183-1198, 1996.

Matter AAG, Ostry DJ. Modifiability of generalization in dynamics learning. J Neurophysiol 98: 3321-3329, 2007.

Mazzoni P, Krakauer JW. An implicit plan overrides an explicit strategy during visuomotor adaptation. J Neurosci 26: 3642-3645, 2006.

Miall RC, Weir DJ, Wolpert DM, Stein JF. Is the cerebellum a Smith predictor? J Mot Behav 25: 203-216, 1993.

Newport R, Brown L, Husain M, Mort D, Jackson SR. The role of the posterior parietal lobe in prism adaptation: failure to adapt to optical prisms in a patient with bilateral damage to posterior parietal cortex. Cortex 42: 720-729, 2006.

Paz R, Boraud T, Natan C, Bergman H, Vaadia E. Preparatory activity in motor cortex reflects learning of local visuomotor skills. Nat Neurosci 6: 882-890, 2003.

Paz R, Vaadia E. Learning-induced improvement in encoding and decoding of specific movement directions by neurons in the primary motor cortex. PLoS Biol 2: E45, 2004.

Poggio T, Bizzi E. Generalization in vision and motor control. Nature 431: $768-774,2004$.

Pouget A, Sejnowski TJ. A new view of hemineglect based on the response properties of parietal neurons. Philos Trans $R$ Soc Lond B Biol Sci 352: 1449-1459, 1997.

Press WH, Flannery BP, Teukolsky SA, Vetterling WT. Numerical Recipes in C. New York: Cambridge Univ. Press, 1992.

Salinas E, Abbott LF. Transfer of coded information from sensory to motor networks. J Neurosci 15: 6461-6474, 1995.

Schwartz AB. Direct cortical representation of drawing. Science 265: 540542, 1994.

Shadmehr R. Generalization as a behavioral window to the neural mechanisms of learning internal models. Hum Mov Sci 23: 543-568, 2004.

Shadmehr R, Krakauer JW. A computational neuroanatomy for motor control. Exp Brain Res 185: 359-381, 2008.

Shumway RH, Stoffer DS. An approach to time series smoothing and forecasting using the EM algorithm. J Time Series Anal 3: 253-264, 1982.

Smith MA, Ghazizadeh A, Shadmehr R. Interacting adaptive processes with different time scales underlie short-term motor learning. PLoS Biol 4: e179, 2006.

Strick PL, Kim CC. Input to primate motor cortex from posterior parietal cortex (area 5). I. Demonstration by retrograde transport. Brain Res 157: 325-330, 1978.

Taylor DM, Tillery SIH, Schwartz AB. Direct cortical control of 3D neuroprosthetic devices. Science 296: 1829-1832, 2002.

Thoroughman KA, Shadmehr R. Learning of action through adaptive combination of motor primitives. Nature 407: 742-747, 2000.

Thoroughman KA, Taylor JA. Rapid reshaping of human motor generalization. J Neurosci 25: 8948-8953, 2005. 
Tseng YW, Diedrichsen J, Krakauer JW, Shadmehr R, Bastian AJ. Sensory prediction errors drive cerebellum-dependent adaptation of reaching. J Neurophysiol 98: 54-62, 2007.

van Beers RJ, Haggard P, Wolpert DM. The role of execution noise in movement variability. J Neurophysiol 91: 1050-1063, 2004.

Wang J, Sainburg RL. Adaptation to visuomotor rotations remaps movement vectors, not final positions. J Neurosci 25: 4024-4030, 2005.
Wise SP, Moody SL, Blomstrom KJ, Mitz AR. Changes in motor cortical activity during visuomotor adaptation. Exp Brain Res 121: 285-299, 1998.

Wolpert DM, Miall RC. Forward models for physiological motor control. Neural Netw 9: 1265-1279, 1996.

Zarzecki P, Strick PL, Asanuma H. Input to primate motor cortex from posterior parietal cortex (area 5). II. Identification by antidromic activation. Brain Res 157: 331-335, 1978. 